\title{
Subliminal Communication in Human-Computer Interaction
}

\author{
Andreas Riener, ${ }^{1}$ Guido Kempter, ${ }^{2}$ Timo Saari, ${ }^{3}$ and Kenneth Revett ${ }^{4}$ \\ ${ }^{1}$ Institute for Pervasive Computing, Johannes Kepler University, Altenberger Strasse 69, 4040 Linz, Austria \\ ${ }^{2}$ UCT Research \& Design Department, University of Applied Sciences Vorarlberg, Hochschulstraße 1, 6850 Dornbirn, Austria \\ ${ }^{3}$ Department of Broadcasting Telecommunications and Mass Media, Temple University, Room 203B Annenberg Hall, Main Campus, \\ 1801 N. Broad Street, Philadelphia, PA 19122, USA \\ ${ }^{4}$ Department for Artificial Intelligence and Interactive Multimedia, Harrow School of Computer Science, University of Westminster, \\ London W1B 2UW, UK
}

Correspondence should be addressed to Andreas Riener, riener@pervasive.jku.at

Received 8 March 2011; Accepted 8 March 2011

Copyright (C) 2011 Andreas Riener et al. This is an open access article distributed under the Creative Commons Attribution License, which permits unrestricted use, distribution, and reproduction in any medium, provided the original work is properly cited.

\section{Introduction}

The research presented in this special issue focuses on subliminal techniques to improve human-computer interaction. Human- and computer-based interactions are ubiquitous in our everyday lives-everything from our mobiles, iPods, to a variety of computer systems creates serious cognitive demands when switching from one to the next. When used simultaneously, extra cognitive demands are placed on us and the likelihood of success is dependent upon our ability to apportion attention to multiple tasks simultaneously. To alleviate the cognitive load associated with interacting with varied computing-based devices, common UI's and modes of operation should be implemented, significantly reducing the amount of information we must store and recall. In addition, subtle cues which could be emitted from the device to assist us during our interactions, prompting us toward the appropriate action required to effectively complete a task. These cues could be delivered in a variety of (communication channels), for instance, visual or auditory cues could be provided according to the specific nature of the current activity.

In order to implement these features, UIs must be provided with the means to detect the nature of the current interaction set, provide the subliminal cues across appropriate channels, and evaluate their success. This approach would benefit from a significant collaborative effort from a range of disciplines: engineering, neuroscience, computer science, psychophysiology, and related fields. The result of this approach provides exciting challenges which will significantly impact society at large, making significant contributions toward a more natural, convenient, and even relaxing future human-computer interface.

The main idea is to "inject" information into the human mind below active awareness, thus transferring supplementary information in a subliminal style without adding load on the cognitive channel. The main benefit of this approach would be the reception of additional, essential information even in the case where (almost) no capacity is left for information transmission in a traditional way. It is anticipated that, for example, events of sudden danger will benefit from using this technology. Nevertheless, the question if subliminal persuasion actually works is still open; however, it has, in case of proven functionality, a potential to reduce cognitive load, stress, or incorrect decisions. This assumption is based on the result of cognitive and social psychologists, who have learnt that stimuli presented subliminally can have a considerable influence over a variety of cognitive processes, possibly even behavior.

Recently, there have been some attempts to extend current user interfaces by means of subliminal communication, with examples being multimedia-driven learning environments, or operating and servicing assistants. However, most user interfaces are still mainly based on symbolic communication. Symbolic communication utilizes a selection of graphical symbols (like icons), symbolic gestures (e.g., pointing movements), or verbal commands. The meaning of such interaction elements might be depending on everyday experience, is defined in style guides, or is given by the 
developer arbitrarily. This results in a distinct code a user has to learn in order to successfully operate the system. In case the symbols used in the interface are not widely spread and understood, a steep learning curve is the consequence.

In contrast to this, subliminal communication requires the integration of spontaneous interaction mechanisms. These mechanisms are characterized by occasionally unexpected actions and results. Therefore, usage of predefined interaction elements is no longer possible in the realm of subliminal communication, because it does not operate on distinguishable semantic units, but rather on continuous transitions among multimodal communication channels. Typical examples of this are natural body movements and nuances in speech of a user as an indicator for specific psychophysiological states, as well as color and shape of graphical user interface elements besides vibro-tactile and acoustic feedback of a system. However, the absence of predefined elements imposes a big challenge on developers who want to implement subliminal communication for human computer interfaces. Efficient human computer interaction has to be ensured, without knowing in advance the relationship of user input and required system actions.

\section{Summary of Submitted Manuscripts}

The special issue at hand received 12 high-quality manuscripts, which have undergone a rigorous peer-review process where the manuscripts were reviewed by two to four reviewers each. In the end, 5 manuscripts were selected for publication in this issue of the AHCI Journal.

In the first paper of this special issue entitled "Benefits of subliminal feedback-loops in human-computer interaction" W. Ritter presents a new approach for adaptive user interfaces whereby analogue signals obtained from the user are deployed to subliminally alter and adapt the interface with the aim of improving some aspect of the user performance and/or experience. Instead of predefined mappings, the used approach is based on an evolutionary feedback-loop. Two presented studies provide support for the proposed solution by confirming significant positive effects regarding performance of users in concentration-intensive games, where the interface or the environment has been adapted in subliminal steps based on physiological signals. In the first study, evolutionary feedback loops adjusted the user interface of a memory game, whereas in the second study the lighting of the test-room was adjusted dynamically. The results show that in settings with an evolutionary feedback-loop test participants were able to reach significantly higher scores compared to the static counterparts. By now, the studies covered short-term effects only (six- and ten-minute game-duration), and longterm effects of such evolutionary feedback-loops were left out and will be investigated in future.

In the paper "Subliminal cues while teaching: HCI technique for enhanced learning" P. Chalfoun and C. Frasson discusses results from an empirical study conducted with a subliminal teaching technique aimed at enhancing learner's performance in intelligent systems through the use of physiological sensors. The applied technique uses carefully designed positive and negative subliminal cues and projects them under the learner's perceptual visual threshold. A positive cue "answer cue" is a hint aiming to enhance the learner's inductive reasoning abilities and projected in a way to help him/her figure out the solution faster but more importantly better. A negative cue "miscue" is also used and aims obviously at the opposite (distract the learner or lead him to the wrong conclusion). The results obtained impressively show that only subliminal cues, not miscues, could significantly increase learner's performance and intuition in a logic-based problem solving task. Nonintrusive physiological sensors (EEG for recording brainwaves, heart rate and skin response to record skin conductivity) were used to record affective and cerebral responses throughout the experiment. The descriptive analysis, combined with the physiological data, provides compelling evidence for the positive impact of answer cues on learning and intuitive decision making in a logic-based problem solving paradigm.

In the third paper of this special issue M. Pasetto and S. O. Barbati have identified that driving is mainly the result of a psychological process that translates data, signals and direct/indirect messages into behavior, which is continuously adapted to the exchange of varying stimuli between man, environment, and vehicle. These stimuli are, however, at times not perceived and at others perceived but not understood by the driver, leading to unsafe behavior of vehicle drivers. In the paper "How the interpretation of drivers' behavior in virtual environment can become a road design tool. A Case Study" they analyzed driver behavior and the dynamic parameters of vehicle motion (i.e., vehicle operating speeds, accelerations and decelerations) after variations in road layout and environmental conditions (e.g., daytime and night driving) for both real driving and virtual environments (i.e., driving simulator). They have demonstrated that the driving simulator is not only a laboratory tool useful for reproducing the trajectory of vehicles in a road environment in a dynamic way, but also an instrument that can forecast drivers' behavior in different situations. During several tests in different scenarios, a statistical comparability has been verified between speeds measured in both the virtual and real environment. In practice, the study puts in evidence when and how the road environment can really be changed in order to become more persuasive for the road users. The virtual environment has the potential to ascertain to what degree this can be done.

In the paper entitled "The sensorium: A multimodal neurofeedback environment", Th. Hinterberger presents a neurofeedback interface that allows people to experience signals from their subliminal psycho-physiological processes visually and auditorily using a thought translation device. In contrast to conventional neurofeedback devices the proposed "Sensorium" focuses on the conscious experiencing of the complex body signals rather than on the self-regulation of a specific component. In the presented pilot study, test subjects have been exposed to their ongoing brain and heart signals. Almost all of them reported an increase in well-being and furthermore, of a widening in their body consciousness.

C. Wolff describe in their paper "A functional driver analyzing concept" an overall system architecture for analyzing 
drowsiness or inattentiveness of the car driver by detecting the driving style of a person. For driving style analysis, they used vehicle data from the CAN-bus only.

\section{Conclusion}

The authors of this special issue show very diverse approaches to an implementation of subliminal communication in human-computer interaction. We believe that it not only illustrates the potential of the application of subliminal techniques for improving human-computer interaction, but also highlights that there are still technical difficulties and unresolved problems limiting a broader deployment in the near future. In the end, we would like to thank all the authors, reviewers as well as the editorial staff of Hindawi for their excellent work during the whole review process.

Andreas Riener Guido Kempter Timo Saari Kenneth Revett 

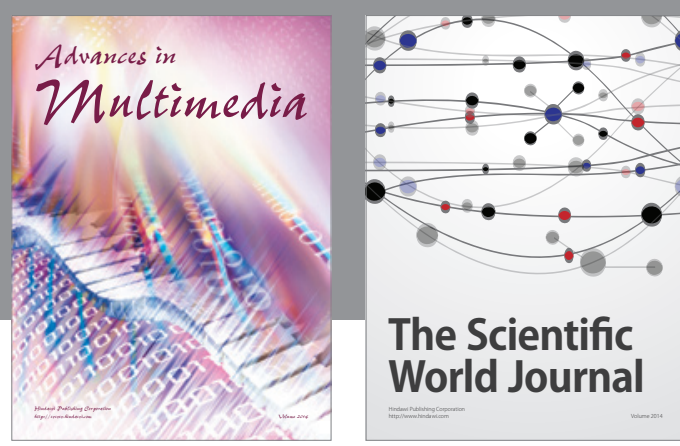

The Scientific World Journal
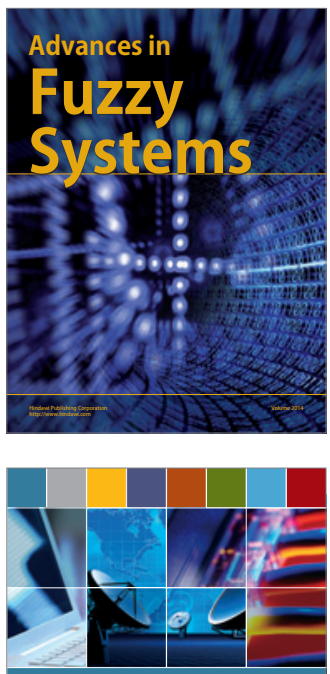

Computer Networks and Communications
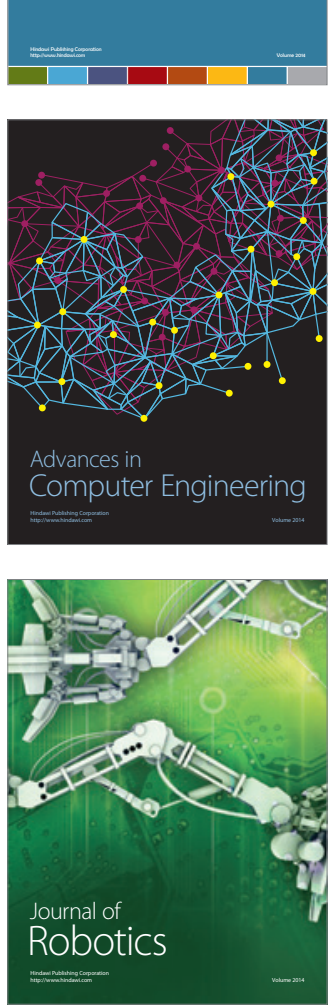
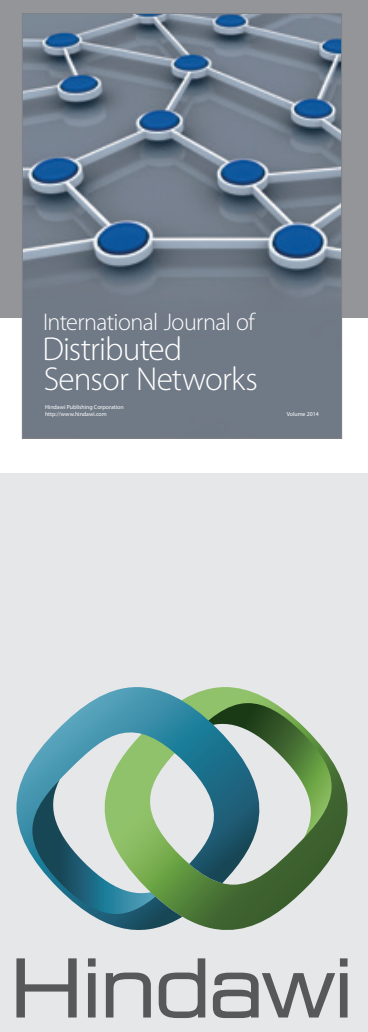

Submit your manuscripts at

http://www.hindawi.com
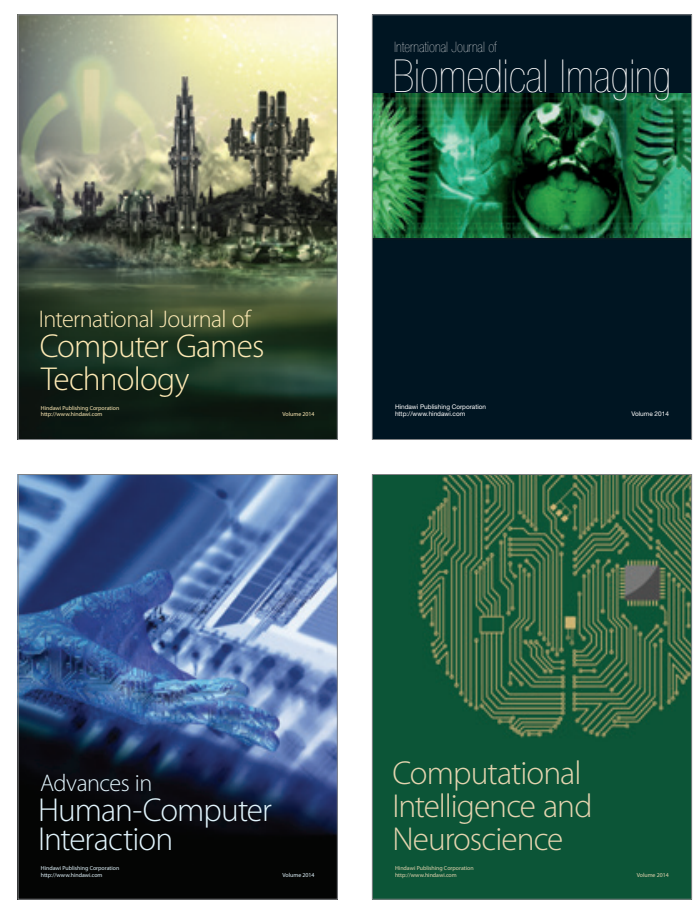
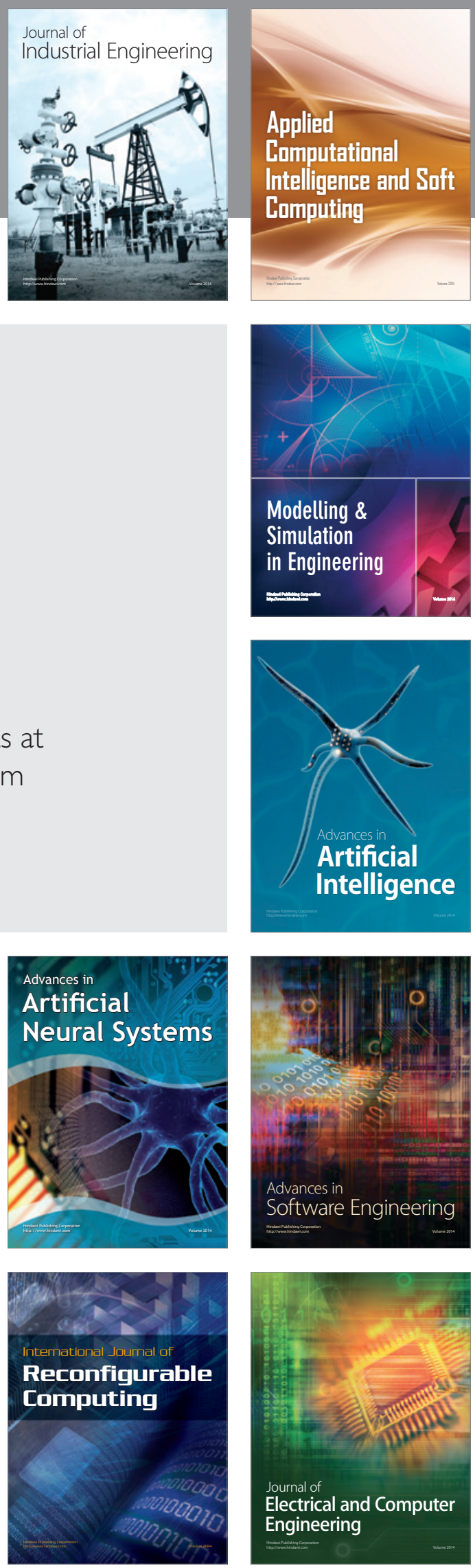